\title{
Multi-Audio Guidance System Using Parametric Speakers, Designed for a Guide Robot
}

\author{
Masashi Ukai, Kenichiro Yasui, Xiuming Wu and Hideo Furuhashi \\ Aichi Institute of Technology1247, Yachigusa, Yakusa-cho, Toyota, JAPAN
}

\begin{abstract}
This study presents a multi-audio guidance system for a guide robot. The system is outfitted with parametric speakers constructed from ultrasonic array transmitters. When the two speakers are closely spaced, their sounds and their ultrasonic waves interact. To investigate these phenomena, the present study demonstrates the system and evaluates its characteristics. Good hearing conditions were obtained when the two parametric speakers were rotated more than $15^{\circ}$ from the "straight-ahead" direction. The audience should stand within $\pm 10^{\circ}$ in front of each speaker. This system could simultaneously guide many persons of different age, nationality, sex, and other demographics with different guide contents.
\end{abstract}

Keyword-Communication robot; parametric speaker; guidance robot; communication robot; directional speaker

\section{INTRODUCTION}

Guidance robots are used in homes, stores, museums, and various other places. Many of these robots are installed with a voice guidance system using a single nondirectional speaker or single wide-directional speakers. The guidance can be heard over a wide area. However, when many people occupy small areas in shops and museums, the guidance should reach only the target audience, without disturbing uninterested persons. Furthermore, interpersonal communication and its contents will depend on the age, sex, nationality, and other characteristics of the listeners. Therefore, the content and language of the guidance should be adapted to different listeners. Simultaneous guidance in Japanese and English improves the experience for English-speaking tourists, whose numbers have increased in recent years. The multi-guidance system is conceptualized in Fig. 1.

A parametric speaker is a directional speaker comprising an ultrasonic array transmitter, that is, a planar arrangement of ultrasonic transmitting elements. The audio sound is modulated by an ultrasonic frequency and transmitted from the array transmitter. The modulated ultrasonic is converted to audio sound while propagating through the air, generating a sound wave with very sharp directivity.

Several previous attempts to include parametric speakers in robots have been reported. The communication robot in [1] can "whisper" during communication and demonstrates good recognition of the user's voice [1]. Another communication robot uses a parametric speaker to detect objects by their reflected sounds [2,3]. Parametric speakers can also direct sound toward the image of a remote user, providing a sense of communication in real time [4]. Previously, we reported faceto-face communication of suitable contents with a specific person, using a guidance robot installed with a parametric speaker and a human vision sensor [5].

The present study demonstrates a multi-audio guidance system with parametric speakers constructed from ultrasonic array transmitters, designed for a guide robot. This study focuses on the system's characteristics.

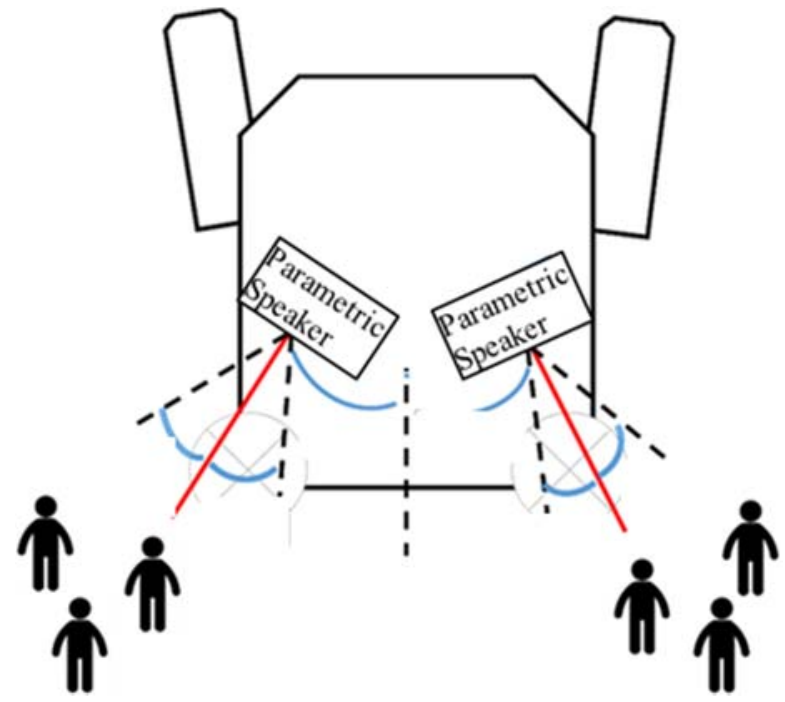

FIGURE I. CONCEPT OF THE MULTI-GUIDANCE ROBOT

\section{THEORY}

A parametric speaker comprises an ultrasonic transmitter array. When the audio signal is modulated by amplitude, the transmitted signal is described as follows.

$$
\begin{array}{r}
f(t)=\left\{1+A_{a} \sin \left(\omega_{a} t\right)\right\} A_{u} \sin \left(\omega_{u} t\right) \\
=A_{u} \sin \left(\omega_{u} t\right)+\frac{A_{a} A_{u}}{2} \cos \left\{\left(\omega_{u}-\omega_{a}\right) t\right\} \\
-\frac{A_{a} A_{u}}{2} \cos \left\{\left(\omega_{u}+\omega_{a}\right) t\right\}
\end{array}
$$

Here, $A_{a}$ and $A_{u}$ are the sound pressures of the audio signal and ultrasonic carrier wave respectively, and $\omega_{a}$ and $\omega_{u}$ are their corresponding angular frequencies. By non-linear effects in the air and interactions between waves of angular frequencies $\omega_{u}$ and $\omega_{u}-\omega_{a}$ or $\omega_{u}$ and $\omega_{u}+\omega_{a}$, the audio 
wave generated by the modulated ultrasonic wave takes the form

$$
g(t)=k A_{u}^{2} A_{a} \sin \left(\omega_{a} t\right)
$$

where $k$ is the conversion efficiency.

However, two modulated signals will interact with each other. The audio signals from two standard audio speakers interact by simple summation, but the interactions between sounds from ultrasonic parametric speakers are complicated by the nonlinear phenomena. A sound wave with anmular frequency $\omega_{a}$ from two parametric speakers is given by

$$
g_{12}(t)=k\left|\dot{A}_{u 1}+\dot{A}_{u 2}\right|^{2} A_{a} \sin \left(\omega_{a} t\right)
$$

Here, $\dot{A}_{u 1}$ and $\dot{A}_{u 2}$ are the sound pressures of the ultrasonic waves (vector) generated by the two parametric speakers. Other harmonic waves are also generated.

\section{Chracteristics OF A Single Parametric Speaker}

The parametric speaker used in this experiment is $10 \mathrm{~cm}$ high and $55 \mathrm{~mm}$ wide and comprises 50 ultrasonic speakers (Fig. 2.). The carrier frequency is $40 \mathrm{kHz}$. The speaker is placed on a guidance robot that communicates face to face with a specific person without disturbing other persons [5]. The speaker is turned in $5^{\circ}$ steps by a turntable. The directivity was measured at $1 \mathrm{~m}$ and $3 \mathrm{~m}$ in front of the speaker. The sound pressure was measured by a $1 / 4$-inch free-field response microphone (46BE, G.R.A.S Sound and Vibration A/S) with a frequency range of 4-80 kHz. The frequency spectrum was analyzed by an FFT analyzer. The usual communication distance between the guidance robot and person is $50 \mathrm{~cm}$ to 5 $\mathrm{m}$. The audio signal is generated in the air as the ultrasonic sound propagates, and the directivity changes with distance. The directivities are plotted in Fig. 3. The audio frequency was $1 \mathrm{kHz}$. In these cases, the audible range $(-6 \mathrm{~dB})$ was approximately $\pm 4^{\circ}$. The horizontal area that the sound can be hear is $15 \mathrm{~cm}$ at $1 \mathrm{~m}$ and $40 \mathrm{~cm}$ at $3 \mathrm{~m}$. The peak sound pressure was approximately $6 \mathrm{~dB}$ lower at $3 \mathrm{~m}$ than at $1 \mathrm{~m}$ from the robot. Despite the reduced sound pressure at the further distance, the total sound power at that distance was boosted by the audio sound, which is generated during propagation in air.

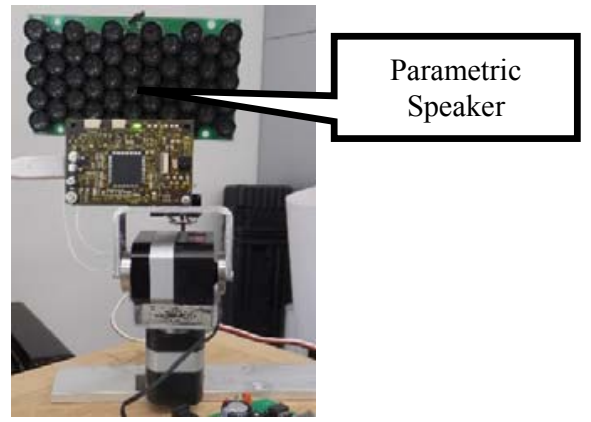

FIGURE II. PARAMETRIC SPEAKER

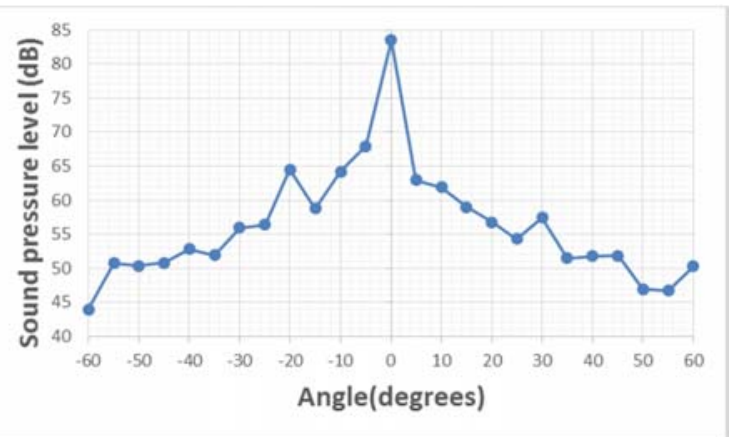

(a) Distance $1 \mathrm{~m}$.

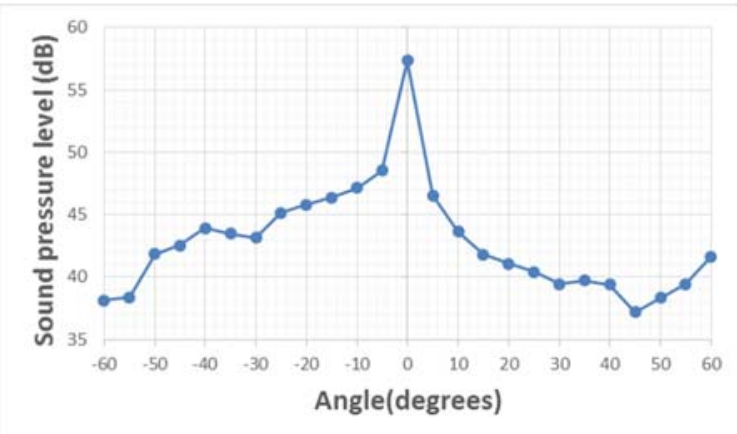

(b) Distance $3 \mathrm{~m}$.

FIGURE III. DIRECTIVITIES OF ONE PARAMETRIC SPEAKER

\section{Characteristics Of The System With Two PARAMETRIC SPEAKERS}

Next, the directivity of the system using two parametric speakers was measured. The experimental setup is shown in Fig. 4. Two speakers placed approximately $30 \mathrm{~cm}$ apart were turned by a $5^{\circ}$-step turntable. The angle of each speaker was changed from 0 to $60^{\circ}$. A $46 \mathrm{BE}$ microphone was placed $3 \mathrm{~m}$ from the speaker system. The audio sound signal was generated by a function generator. To avoid interference between the audio signals generated by the two signals, one speaker was supplied with a $1050 \mathrm{~Hz}$ signal, the other with a $950 \mathrm{~Hz}$ signal. The frequency spectrum was analyzed by an FFT analyzer. Figure 5 plots the sound pressure levels as functions of speaker angle from $0^{\circ}$ to $60^{\circ}$. The audio sounds did not overlap at angles over $15^{\circ}$. The minimum distance of sound clarity in the two listening areas was $50 \mathrm{~cm}$ and $1.6 \mathrm{~m}$ at distances of $1 \mathrm{~m}$ and $3 \mathrm{~m}$ from the robot, respectively.

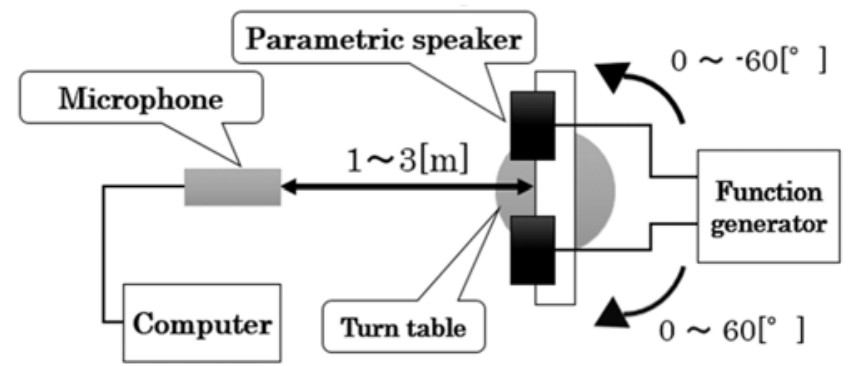

FIGURE IV. SET-UP OF DIRECTIVITY EXPERIMENT USING TWO PARAMETRIC SPEAKERS 


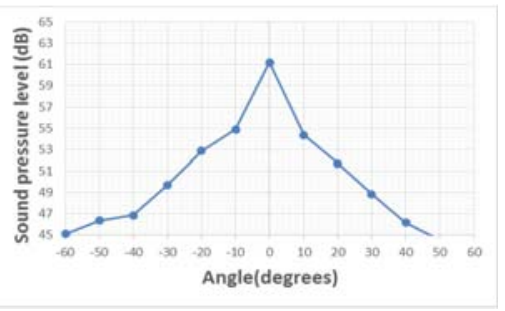

(a) Speaker angle $=0^{\circ}$.

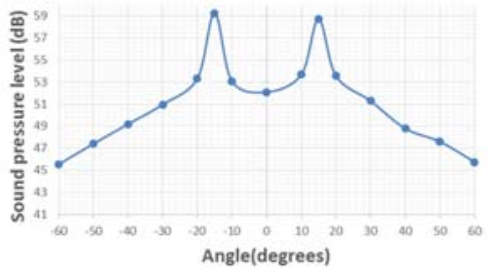

Speaker angle $=15^{\circ}$

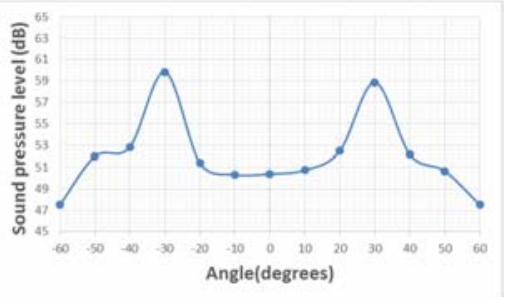

(b) Speaker angle $=30^{\circ}$.

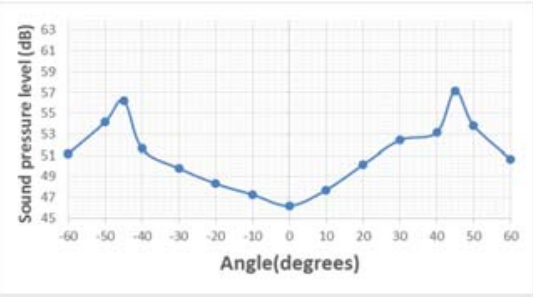

(c) Speaker angle $=45^{\circ}$

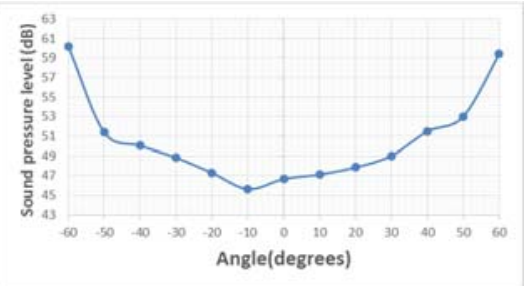

(d) Speaker angle $=60^{\circ}$

FIGURE V. DIRECTIVITIES OF THE SYSTEM WITH TWO PARAMETRIC SPEAKERS POSITIONED AT DIFFERENT ANGLES RELATIVE TO THE MICROPHONE.

Finally, the angle of the two areas in which listeners can properly hear the guidance was studied. In this experiment, two parametric speakers produced Japanese and English guidance with the same sound pressure. The angle of the speakers was changed from $0^{\circ}$ to $90^{\circ}$, and the sound was evaluated by five human subjects. The sound was rated on the 5 -point scale shown in Table 1.
Figures 6 shows the evaluation results. Although the perceived sound quality differed among the subjects, a similar tendency was obtained. Figure 7 shows the evaluation results when subjects stood in front of the speaker. The guidance in each area was easily heard when the speaker was rotated more than $15^{\circ}$ (average evaluation score $>4$ ). The minimum distances of sound clarity in the two listening areas were 53 $\mathrm{cm}$ and $1.6 \mathrm{~m}$ at $1 \mathrm{~m}$ and $3 \mathrm{~m}$ from the robot, respectively. The width of the area of sound clarity was $\pm 10^{\circ}$ in front of the speaker, as shown in Fig. 8, corresponding to $35 \mathrm{~cm}$ at $1 \mathrm{~m}$ and $1.1 \mathrm{~m}$ at $3 \mathrm{~m}$.

TABLE I. EVALUATION RATING OF THE SOUND

\begin{tabular}{|l|l|}
\hline \multicolumn{2}{|c|}{ Evaluation scoring } \\
\hline 5 & Can hear clearly \\
\hline 4 & Can hear \\
\hline 3 & Can hear faintly \\
\hline 2 & Difficult to hear \\
\hline 1 & Unable to hear \\
\hline
\end{tabular}

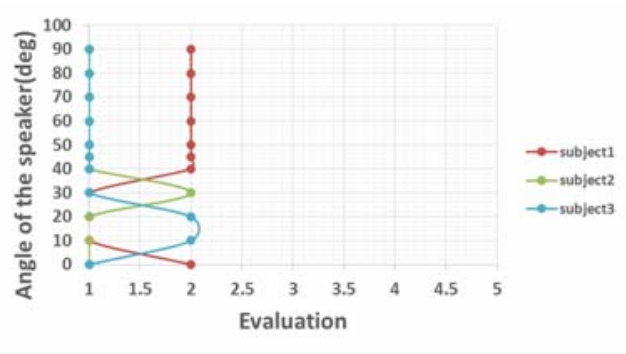

(a) Speaker direction $=0^{\circ}$.

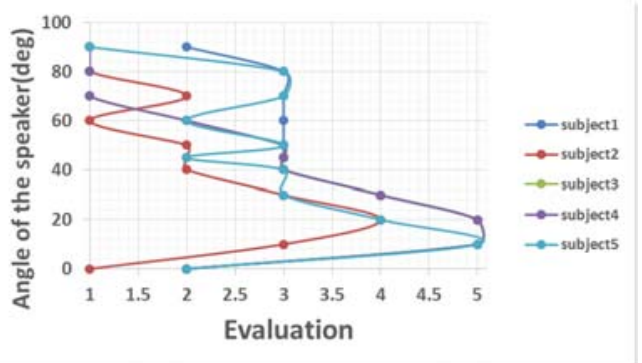

(b) Speaker direction $=22.5^{\circ}$.

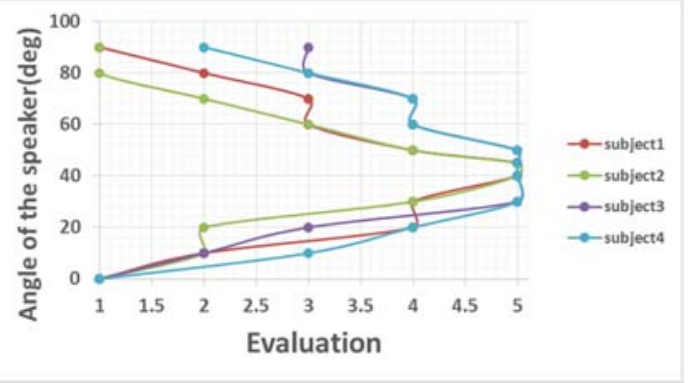

(c) Speaker angle $=45^{\circ}$. 


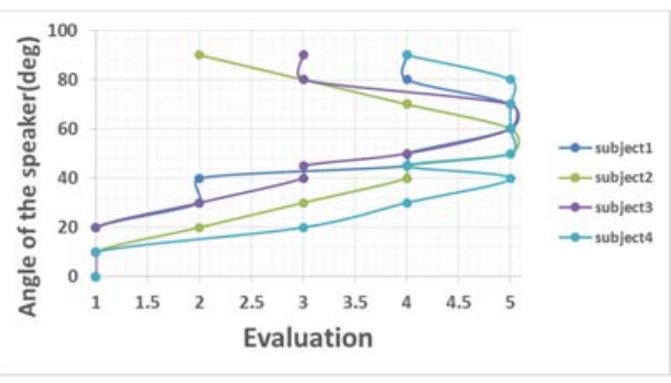

(d) Speaker angle $=67.5^{\circ}$.

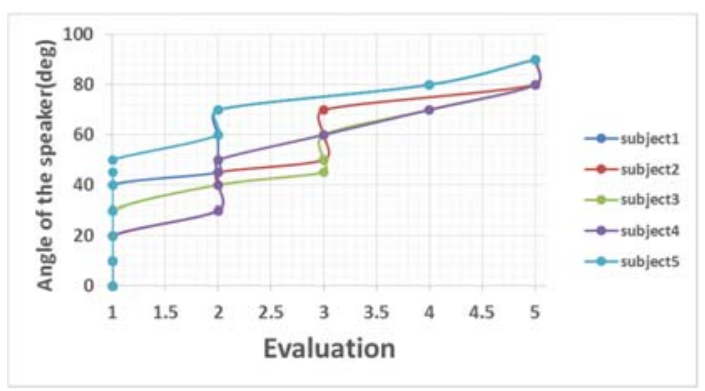

(e) Speaker angle $=90^{\circ}$.

FIGURE VI. SOUND EVALUATION BY HUMAN LISTENERS FOR DIFFERENT DIRECTIONS OF THE SPEAKERS

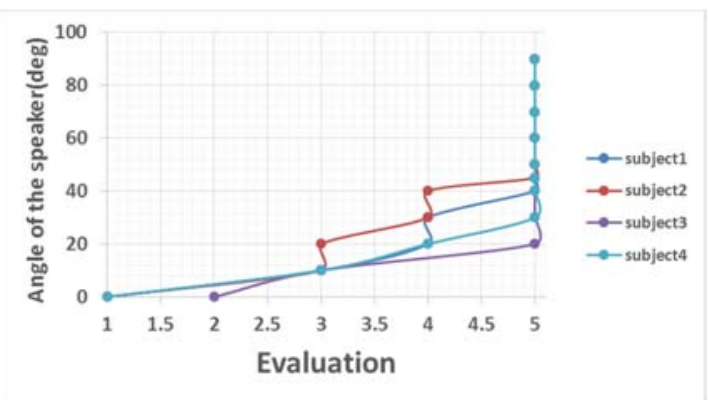

FIGURE VII. SOUND EVALUATION BY HUMAN LISTENERS STANDING IN FRONT OF THE SPEAKER

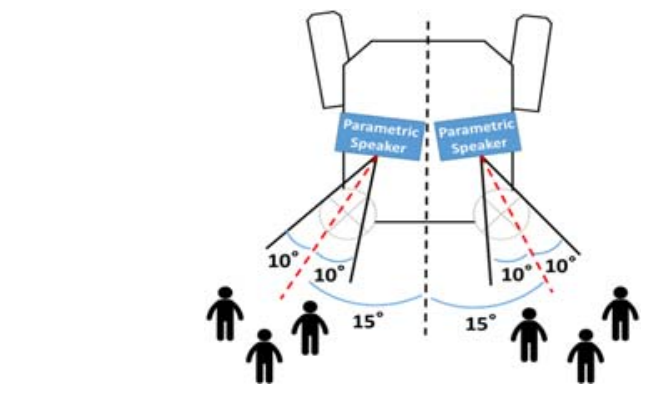

FIGURE VIII. MOUNTING POSITION OF THE TWO DIRECTIONAL SPEAKERS AND POSITIONS OF THE AUDIENCE

\section{CONCLUSION}

A multi audio guidance system based on parametric speakers was designed for a guide robot. The detailed characteristics of the system were studied. Good hearing conditions were obtained when the two parametric speakers were oriented at more than $15^{\circ}$ relative to the straight-ahead direction. The area of best sound quality was $\pm 10^{\circ}$ in front of each speaker.

This system could simultaneously provide different guide contents to many persons of different age, nationality, sex, and other demographics. Next, we will investigate the characteristics of the system under other conditions such as different parametric speaker configurations and conduct theoretical investigations by simulating the audio sound generations of two interacting parametric speakers.

\section{ACKNOWLEDGMENT}

This study has received a grant of JSPS Research Institute expenses $15 \mathrm{~K} 05879$.

\section{REFERENCES}

[1] Kazuhiro Nakadai "Towards Information Integration for Human-Robot Interaction" The Institute Of Electronics, Information And Communication engineers (IEICE), pp37-44 (2006).

[2] Yukiko Yamamoto, Kentatro Ishii, Kazuhiro Nakadai, Michita Imai "An Object Reference system Using Directional Speaker" Human Interface Symposium, pp303-308 (2006).

[3] Koji Kainou, Hotaka Takizawwa, Mayumi Aoyagi, Nobuo Ezaki, Shinji Mizuno "Basic Study on Remote ADL Training for the Visually Impaired Based on a Parametric-speaker-equipped Robot and Its Evaluation" FIT, pp429-430 (2014).

[4] Ryota Fujimura, Guo Bin, Ren Ohmura, Michita Imai "Remy: A remote communication system that refers real world objects" GN Workshop, pp85-90 (2008).

[5] Kenichiro Yasui, Ukai Masashi, Wei Quan, Xiuming Wu, Hideo Furuhashi, "One-to-one Audio Guidance System Using Human Vision, Designed for a Guide Robot," The 2015 International Conference on Computational Science and Computational Intelligence, IEEE Xplore pp. 841-842,(2015) 